$$
(x+y)^{p} \equiv x^{p}+y^{p} \bmod p^{3},
$$

from which $\left(1^{\prime}\right)$ is readily deduced.

Professor Birkhoff points out further that the test fails to be effective for all primes $p$ of the form $6 n+1$. For if $p=6 n+1$ it follows from the theory of primitive roots modulo $p^{3}$ that the congruence

$$
t^{3} \equiv 1 \bmod p^{3}
$$

has a solution $t$ for which $t-1$ is prime to $p$. Hence also

Then we have

$$
t^{2}+t+1 \equiv 0 \bmod p^{3} \text {. }
$$

$(t+1)^{p}=(t+1)(t+1)^{6 n} \equiv(t+1)\left(-t^{2}\right)^{6 n} \equiv t+1 \bmod p^{3}$, $(t+1)^{p^{2}} \equiv(t+1)^{p} \equiv t+1 \bmod p^{3}$,

and

$$
t^{p} \equiv t \cdot t^{6 n} \equiv t \bmod p^{3}, \quad p^{2} \equiv t^{p} \equiv t \bmod p^{3} .
$$

Therefore

Now put

$$
(t+1)^{p^{2}} \equiv t^{p^{2}}+1 \bmod p^{3} .
$$

Then

$$
t=\sigma+v p, \quad(0<\sigma<p-1) .
$$

$$
t^{p^{2}} \equiv \sigma^{p^{2}}, \quad(t+1)^{p^{2}} \equiv(\sigma+1)^{p^{2}} \bmod p^{3} .
$$

Therefore

$$
(\sigma+1)^{p^{2}} \equiv \sigma^{p^{2}}+1 \bmod p^{3}, \quad(0<\sigma<p-1) .
$$

This is relation (7) of my previous note; from this follows (1) as in the earlier treatment. Hence (1) is satisfied by all primes of the form $6 n+1$. Therefore the test can be useful only when the exponent $p$ is 3 or is of the form $6 n-1$.

INDIANA UNIVERSITY, March, 1913.

\title{
AN EXTENSION OF A THEOREM OF PAINLEVÉ.
}

BY DR. E. H. TAYLOR.

(Read before the American Mathematical Society, October 26, 1912.)

Theorem: Let $f(z)$ be a function which is single-valued and analytic throughout the interior of a region $S$ of the $z$-plane, $z=x+y i$. If $f(z)$ vanishes at every point of a 
connected portion of the boundary of $S$, two points of which, $A$ and $B$, can be joined by a curve $C$ lying wholly within $S$, then $f(z) \equiv 0$.

Painlevé* has proved this theorem for the case where the portion of the boundary in question is an arc of a regular curve. The object of the present paper is to show that the theorem is true in the general case for which the theorem is above stated; for example, when in every neighborhood of any point of the boundary there are points that cannot be approached along a continuous curve lying in the region.

Denote by $\Sigma$ the region bounded completely by $C$ and the portion $A B$ of the boundary along which $f(z)$ vanishes. We will assume that $\Sigma$ lies within a circle of radius unity with center at $O:(z=0)$, a point of $A B$, but which is distinct from both $A$ and $B$.

Let the z-plane be transformed by

$$
z_{1}=\log z \text {. }
$$

The interior of the unit circle is thus mapped on the half-plane $x_{1}<0, \Sigma$ going over into a region $\Sigma_{1}$ of that half-plane which extends to infinity and has the point $z_{1}=0$ as an exterior point.

We will next apply the transformation

$$
z_{2}=-i / z_{1} \text {. }
$$

The interior of $\Sigma_{1}$ is thereby carried over into the interior of a region $\Sigma_{2}$ which lies in the upper half of the $z_{2}$-plane. Thus $\Sigma$ has been mapped on $\Sigma_{2}$, the boundary point $O:(z=0)$ of $\Sigma$ going over into the boundary point $z_{2}=0$ of $\Sigma_{2}$.

As a third transformation we will use

$$
z_{3}=\sqrt{z_{2}}, \quad z_{3}=r_{3} e^{\phi_{3} i}
$$

which makes the image $\Sigma_{3}$ of $\Sigma$ lie within the region $0<\varphi_{3}<\pi / 2$, as indicated in the figure. We will denote the images in the $z_{3}$-plane of $O, A, B$, and $C$ by $O_{3}, A_{3}, B_{3}$, and $C_{3}$, respectively.

There is a neighborhood of $O$ in the original region $\Sigma$ that contains no boundary point of $\Sigma$ in which $f(z)$ does not vanish; in particular, this neighborhood contains no point of $C$.

* Toulouse Annales, vol. 2 (1888), p. B 29. 
Therefore, with $O_{3}$ as center, it is possible to construct a circumference $K$ outside of which $C_{3}$ will lie, and which will cut off from $\Sigma_{3}$ one or more regions. One of these regions $\Sigma_{3}{ }^{\prime}$ will

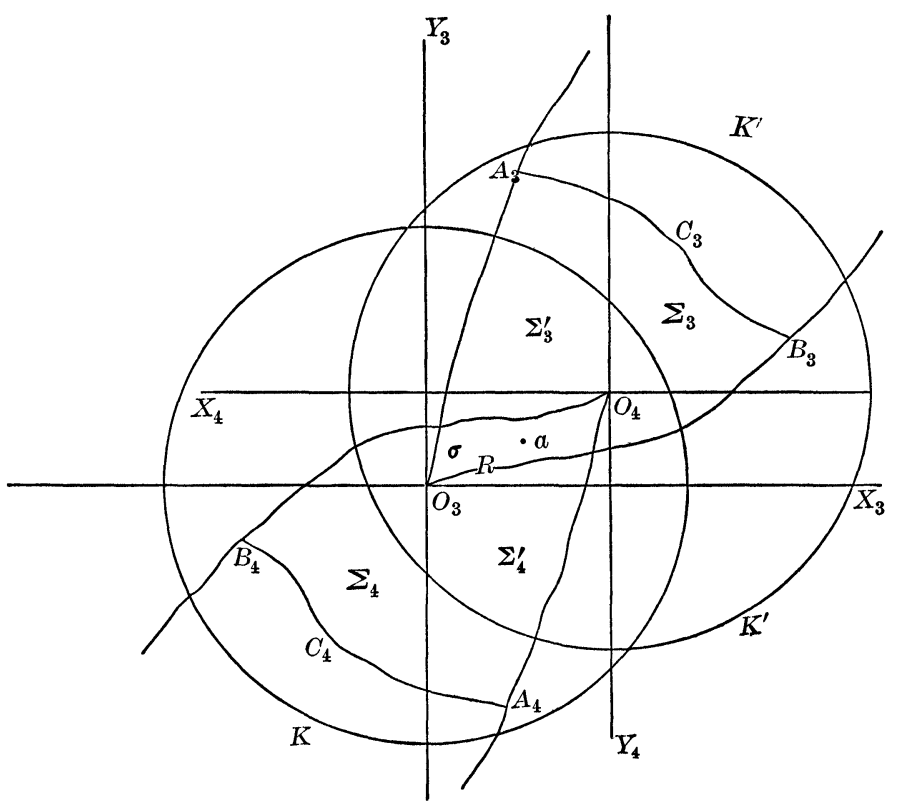

contain a point $a$ in its interior whose distance from $O_{3}$ is less than half the radius of $K$.

Next, rotate the plane about the point $a$ as a center by the transformation

$$
z_{4}=-z_{3}+2 a .
$$

This transforms the axes of reals and pure imaginaries into lines parallel to them, and bounding with them a rectangle $R$.

Let the images in the $z_{4}$-plane of $\Sigma_{3}{ }^{\prime}$ and $K$ be called $\Sigma_{4}{ }^{\prime}$ and $K^{\prime}$. The regions $\Sigma_{3}{ }^{\prime}$ and $\Sigma_{4}{ }^{\prime}$ have one or more regions in common, all of which lie in $R$, and hence in the region common to $K$ and $K^{\prime}$. Let $\sigma$ denote one of these regions. From the method of constructing $K$ it follows that $K$, and hence $K^{\prime}$, contain the images of no boundary points of $\Sigma$ except those in which $f(z)$ vanishes. Consequently every boundary point of $\sigma$ is the image of a boundary point of $\Sigma$ in which $f(z)$ vanishes.

Let the function into which $f(z)$ is carried by the transfor- 
mations (1), (2), and (3) be denoted by $\varphi\left(z_{3}\right)$. The latter function is transformed by (4) into $\varphi\left(-z_{3}+2 a\right)$. From the hypotheses of the theorem and the properties of the transformations employed, it follows that the function

$$
\varphi\left(z_{3}\right) \cdot \varphi\left(-z_{3}+2 a\right)
$$

is analytic throughout the interior of $\sigma$ and vanishes at every point of the boundary. Hence both the real and the pure imaginary parts of this function vanish at every point of the boundary of $\sigma$ and are, therefore, both identically zero, since a function that is single-valued and harmonic throughout the interior of a region and vanishes at every point of the boundary is identically zero.* Since

$$
\varphi\left(z_{3}\right) \cdot \varphi\left(-z_{3}+2 a\right) \equiv 0,
$$

one of the factors vanishes identically, and therefore

$$
f(z) \equiv 0 \text {. }
$$

\section{MATHEMATICAL PHYSICS AND INTEGRAL EQUATIONS.}

Die Integralgleichungen und ihre Anwendungen in der mathematischen Physik. Vorlesungen an der Universität zu Breslau, gehalten von Adolf Kneser. Braunschweig, Vieweg, 1911. 8vo. viii +243 pp.

THE solution of various boundary value problems for a partial differential equation by means of the expansion of an arbitrary function in series of solutions of ordinary differential equations involving a parameter constitutes one of the most important applications of the theory of integral equations. Here, as so often elsewhere, mathematical physics has first propounded the question, and it has been the task of analysis to furnish the answer. Especially close, therefore, has been the connection between mathematical physics and integral equations; especially interesting must be likewise a method of treatment which aims to exhibit this connection as vividly as possible. Such is the method of Kneser's book; we learn

\footnotetext{
* Osgood, Lehrbuch der Funktionentheorie, vol. 1, 2d ed., 1912, p. 623.
} 